The University of Southern Mississippi

The Aquila Digital Community

Faculty Publications

10-13-1997

\title{
Nonuniversal Scaling and Conformational Crossover of Polymer Chains in an Electrophoretic Deposition
}

Grace M. Foo

National University of Singapore

Ras B. Pandey

University of Southern Mississippi, ras.pandey@usm.edu

Follow this and additional works at: https://aquila.usm.edu/fac_pubs

Part of the Physics Commons

\section{Recommended Citation}

Foo, G. M., Pandey, R. B. (1997). Nonuniversal Scaling and Conformational Crossover of Polymer Chains in an Electrophoretic Deposition. Physical Review Letters, 79(15), 2903-2906.

Available at: https://aquila.usm.edu/fac_pubs/5434

This Article is brought to you for free and open access by The Aquila Digital Community. It has been accepted for inclusion in Faculty Publications by an authorized administrator of The Aquila Digital Community. For more information, please contact Joshua.Cromwell@usm.edu. 


\title{
Nonuniversal Scaling and Conformational Crossover of Polymer Chains in an Electrophoretic Deposition
}

\author{
Grace M. Foo ${ }^{1}$ and R. B. Pandey ${ }^{2}$ \\ ${ }^{1}$ Supercomputing and Visualization Unit, National University of Singapore, Singapore 119260 \\ ${ }^{2}$ Department of Physics and Astronomy, Program in Scientific Computing, University of Southern Mississippi, \\ Hattiesburg, Mississippi 39406-5046
}

(Received 26 June 1997)

\begin{abstract}
A computer simulation model of electrodeposition of polymer chains on an impenetrable wall is used to evaluate the power-law scaling exponents $\left(\nu_{x(y)}\right)$ for the longitudinal and transverse spread, $R_{g x(y)} \sim L_{c}^{\nu_{x(y)}}$; we find that the exponents $\nu_{x(y)}$ depend on the field strength, i.e., they are nonuniversal. A conformational crossover is observed for the transverse spread from the bulk with $\nu_{y} \simeq 1 / 3-2 / 3$ to the wall with $\nu_{y} \simeq 2 / 3-1$. A similar crossover also occurs for the longitudinal component of $R_{g}$ but with an opposite trend, i.e., magnitude of $\nu_{x}$ is larger in bulk than at the wall. [S0031-9007(97)04202-6]
\end{abstract}

PACS numbers: 61.41.+e, 81.15.Pq

Dynamics of interfacial growth and scaling described by the Kardar-Parisi-Zhang (KPZ) model [1] and its connection to scaling in directed polymers [2] has opened up enormous possibilities for addressing interesting questions in a variety of systems for about a decade [3-5]. Kardar and Zhang [2] made a fundamental contribution to the scaling of transverse fluctuations with the length of directed polymers subject to external impurities, i.e., polyelectrolytes in a gel matrix. They argue that a configuration of directed polymer can be regarded as the world of line. Therefore, a path integral representation of the overall Boltzmann weight $W\left(R_{y}, N\right)$ can be used to describe the transverse fluctuation $R_{g y}$ ( $y$ component of the radius of gyration) with the length $N$ of the $x$-directed polymer, i.e.,

$$
W\left(R_{y}, N\right)=\int_{(0,0)}^{\left(R_{y}, N\right)} D R_{y}^{\prime}\left(N^{\prime}\right) \exp \left\{-\int_{0}^{N} d N^{\prime}\left[(\gamma / 2)\left(d R_{y}^{\prime} / d N\right)^{2}+\mu\left(R_{y}^{\prime}, N^{\prime}\right)\right]\right\},
$$

where $\gamma$ is a bare line tension, and $\mu\left(R_{y}, N\right)$ is an impurity term with fluctuation $\left\langle\mu(x, N) \mu\left(x^{\prime}, N^{\prime}\right)\right\rangle=\sigma^{2} \delta(N-$ $\left.N^{\prime}\right) \delta^{d-1}\left(R_{y}-R_{y}^{\prime}\right)$ in $d$-dimensional space. In two dimensions, with the impurities, the scaling exponent $\nu$ for the transverse fluctuation with the length $R_{y} \sim N^{\nu_{y}}, \nu_{y}=$ $2 / 3$ exactly. Mapping Eq. (1) to the randomly stirred Burgers equation and using dynamical renormalization group method, and by numerical simulations in $d \geq 2$, Kardar and Zhang predicted $[2,6,7]$ that the scaling exponent $\nu_{y}$ is superuniversal with $\nu_{y}=2 / 3$ in $d=2,3$, and 4.

We consider a computer simulation model for electrodeposition of polymer chains [8] to study the universality of scaling exponent of the chains [2]. Further we address the following more general question: How does the conformation of chain change from bulk to the surface [9$11]$ as a function of electric field $(B)$ and temperature $(T)$ ? We find that the scaling exponent of the transverse (longitudinal) spread of the polymer chains at the wall (bulk) is nonuniversal as it depends on the field strength $(B)$ and temperature and that its magnitude for the chains at the wall differs substantially from that in the bulk. Studying the conformation of chains in such a model could be useful in understanding the stability and interfacial sliding of polymers [12-16] and friction in various applications including lubrications, spray painting, and coating under pressure.
Electrophoretic flow of polymers through a gel matrix, i.e., a gel electrophoresis [17-24] is another example where the knowledge of chains conformations as they deposit on a wall (pore boundary of gel) could be useful in understanding and assessing the errors in such applications as DNA fingerprinting.

We consider a discrete lattice of size $L_{x} \times L_{y}$ with large aspect ratios $\left(L_{x} / L_{y}\right) ; L_{x}=1000, L_{y}=200$ is used for most of our data but different lattice sizes are used to check for the severe finite size effects. Polymer chains [each of length $L_{c}$ with $\left(L_{c}+1\right)$ nodes connected by a constant bond of unit length] are released at about a constant rate from one end (source end $x=1$ ) of the sample and are deposited at impenetrable wall at the opposite end $\left(x=L_{x}\right)$ via "slithering snake" (reptation dynamics). In addition to excluded volume, a nearest neighbor polymerpolymer repulsive, and polymer-wall attractive (adsorbing) interactions are considered [8]. The external field $B$ is coupled with the movements of chain nodes via interaction energy $-B \Delta X$, where $\Delta X(=0$ or \pm 1$)$ is the displacement of a node along the $x$ direction. Metropolis algorithm is used to reptate the chains. An attempt to move chains $L_{x} \times L_{y}$ times is defined as unit Monte Carlo step (MCS). Profiles of polymer density and radius of gyrations are studied in detail. The simulation is carried out for a sufficiently long time to obtain stable profiles at the wall 


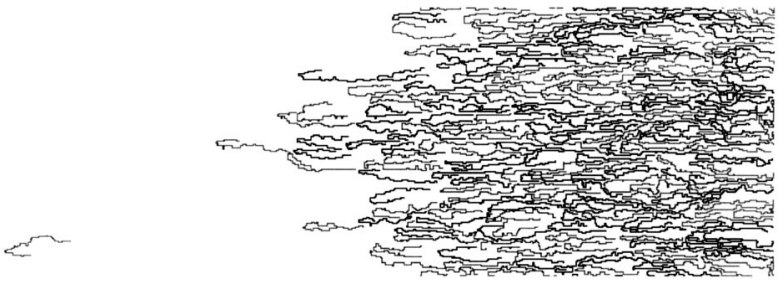

FIG. 1. Snapshot of chains of length $L_{c}=120$ at $T=1$ and $B=2.0$.

and around in the bulk. We use a sufficiently large number of independent runs to estimate the average quantities reliably.

Figure 1 shows a typical snapshot of the instantaneous chain configurations. We see that a sufficiently large concentration of polymer chains has already evolved at the wall extending into a considerable fraction of neighboring layers in the bulk. A typical profile of monomer density and radius of gyration as a function of $x$ is presented in Fig. 2. The density profile suggests that it has reached the stable value at the wall and that it decreases as we go into the bulk ( $800 \leq x<L_{x}$ ); the decay of density away from the wall is primarily due to steric screening and mainly due to entropic contribution of free energy. Various interesting

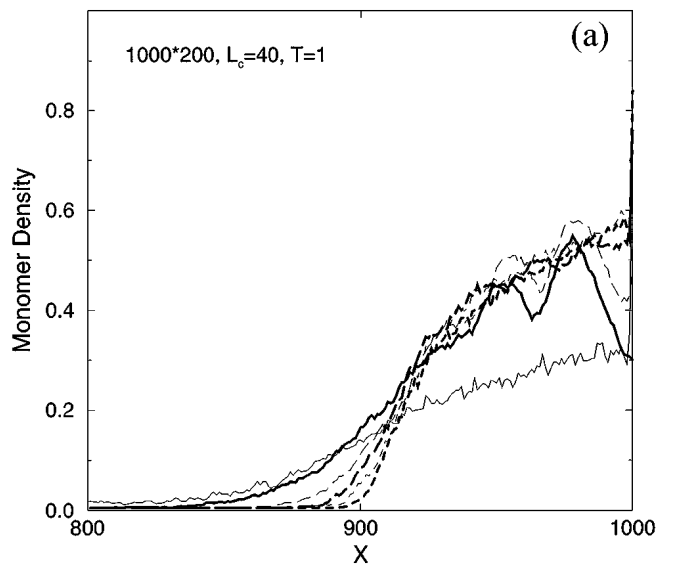

$$
\begin{aligned}
-B & =0.1 \\
---B & =0.5 \\
--B & =1.0 \\
--B & =2.0 \\
---B & =5.0 \\
-B & =10.0
\end{aligned}
$$
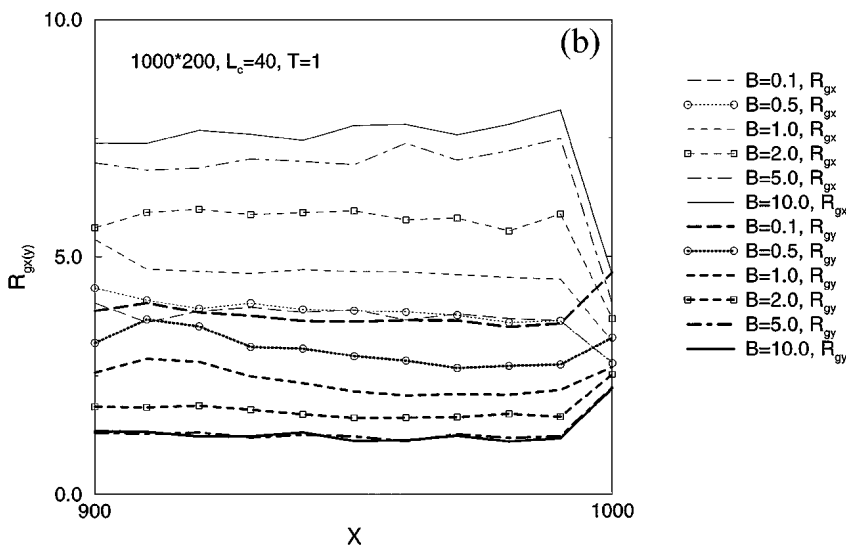

FIG. 2. Profiles along $x$ direction of (a) monomer density and (b) radius of gyration for chains of length $L_{c}=40$ at $T=1$. Lattice size $1000 \times 200$ with 20 independent samples is used. effects such as the onset of oscillations near the wall and scaling of polymer density with the chain length and effects of field has been described in detail elsewhere [8]. The profile of the radius of gyration exhibits a contrast in variation of its $x$ and $y$ components near the wall: while $R_{g x}$ decays with $x$ from bulk to wall, $R_{g y}$ increases, and the rate of variation is enhanced on increasing the field.

In order to evaluate the scaling exponents $\nu_{x}$ and $\nu_{y}$ for the longitudinal $\left(R_{g x} \sim L_{c}^{\nu_{x}}\right)$ and transverse $\left(R_{g y} \sim\right.$ $\left.L_{c}^{\nu_{y}}\right)$ components of the radius of gyration $\left(R_{g} \sim L_{c}^{\nu}\right)$, we
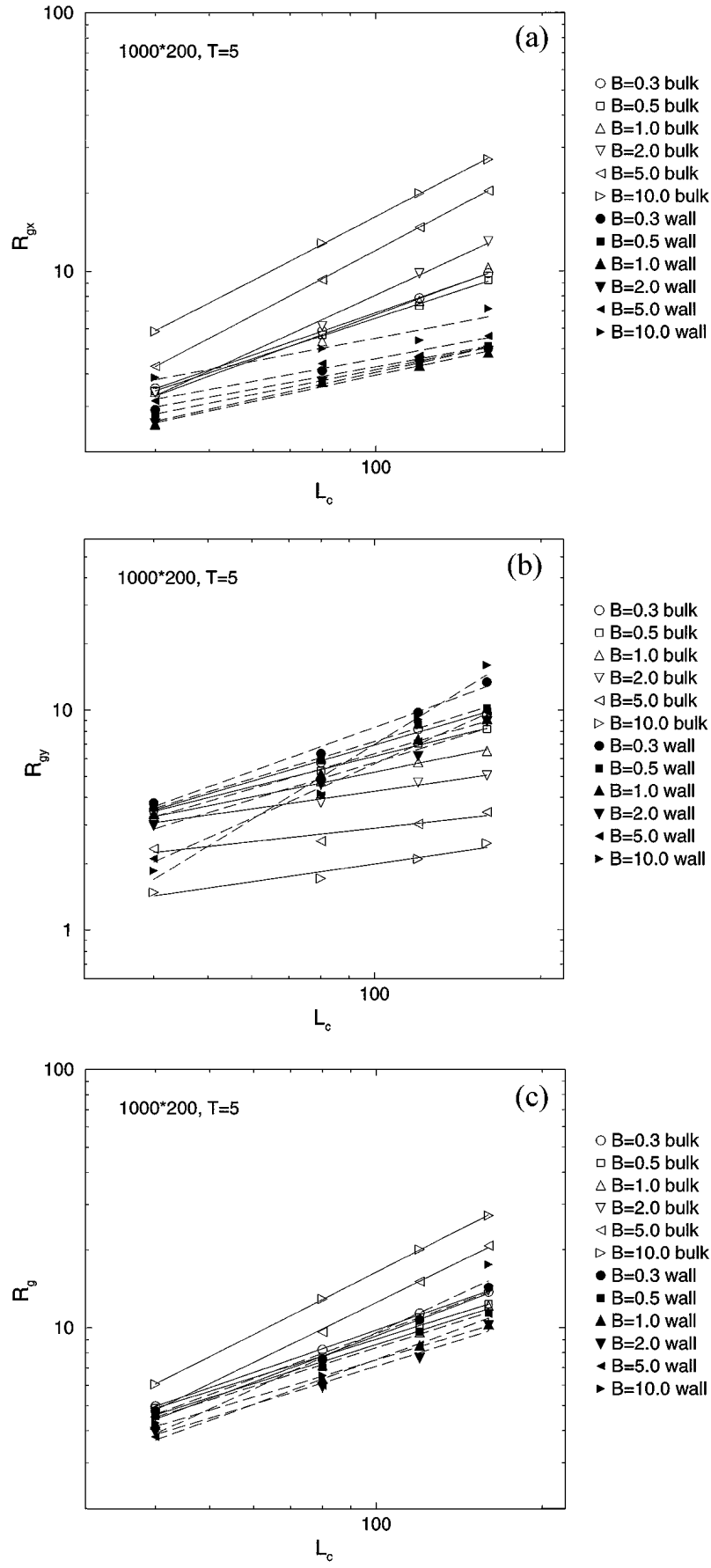

FIG. 3. A typical (a) $R_{g x}$, (b) $R_{g y}$, and (c) $R_{g}$ versus $L_{c}$ plot for chains at $T=5$, with the same statistics as in Fig. 2. 




FIG. 4. Exponent $\nu$ versus $B$ for chains at $T=10$.

examine their variation with the chain length on a log$\log$ scale for chain at the wall and in the bulk. A typical variation is shown in Fig. 3 for various values of field strength. The linear fit seems consistent with the power- law scaling at least with a leading exponent. The longitudinal component in the bulk shows that the exponent $\nu_{x}$ depends on the field strength; a variation of the exponent with field is presented in Fig. 4. It is rather difficult to provide a closed form empirical expression based on our data, nevertheless, a nonmonotonic dependence could be speculated from a close inspection. For example, at the temperature $T=10.0, \nu_{x}$ increases $\left(\nu_{x}=0.7-1.0\right)$ on increasing the field in low field regime $(B=1.0-5.0)$ before it saturates to its maximum value $\left(\nu_{x}=1.0\right)$ at high field strength $(B \geq 5.0)$; at $B \leq 1.0, \nu_{x}$ shows an increasing trend with decreasing field leading to a minimum at $B \simeq 1.0$. The longitudinal component $\left(R_{g x}\right)$ at the wall also seems to suggest a power-law scaling but with much lower exponent values $\left(\nu_{x} \simeq 0.3-0.4\right.$ at $B=0.5-5.0$, $T=10.0$ ); the magnitude of the exponent is less sensitive to field strength.

The transverse spread of the chain, described by $R_{g y}$, also exhibits a power-law scaling both in the bulk as well as at the wall [see Fig. 3(b)]. The nature of its variation

TABLE I. Exponent $\nu$ in $R_{g} \sim L_{c}^{\nu}$. $x$ and $y$ components of $R_{g}$ are described by exponents $\nu_{x}$ and $\nu_{y}$, respectively. The error bar $\Delta \nu$ in evaluating the exponents varies. $\Delta \nu \sim$ $0.07-0.10$ for the chains in the bulk. There are considerably larger error bars (as large as $\sim 0.15$ ) for the chains at the wall due to poor statistics; many data points do not fit the power-law dependence indicated by - . The value of exponents slightly larger than 1 are truncated to 1 and exponents with relatively larger error bar are identified by $*$.

\begin{tabular}{|c|c|c|c|c|c|c|c|}
\hline \multirow{2}{*}{$\begin{array}{c}\text { Temperature } \\
T\end{array}$} & \multirow{2}{*}{$\underset{B}{\mathrm{Bias}}$} & \multicolumn{3}{|c|}{ Bulk } & \multicolumn{3}{|c|}{ Wall } \\
\hline & & $\nu$ & $\nu_{x}$ & $\nu_{y}$ & $\nu$ & $\nu_{x}$ & $\nu_{y}$ \\
\hline 100.0 & 5.0 & 0.73 & 0.68 & 0.77 & 0.87 & 0.38 & $1.00 *$ \\
\hline 100.0 & 10.0 & 0.69 & 0.72 & 0.65 & 0.70 & 0.36 & 0.82 \\
\hline 10.0 & 0.3 & 0.77 & 0.75 & $0.77 *$ & 0.88 & 0.37 & 1.0 \\
\hline 10.0 & 0.5 & 0.74 & 0.77 & 0.70 & 0.75 & 0.32 & 0.89 \\
\hline 10.0 & 1.0 & 0.68 & 0.69 & 0.67 & 0.70 & 0.39 & 0.81 \\
\hline 10.0 & 2.0 & 0.67 & 0.76 & 0.54 & 0.64 & 0.41 & 0.74 \\
\hline 10.0 & 5.0 & 0.84 & 1.00 & 0.33 & 0.65 & 0.50 & 0.75 \\
\hline 10.0 & 10.0 & $1.00^{*}$ & $1.00 *$ & 0.26 & 0.76 & $0.43 *$ & $1.00 *$ \\
\hline 5.0 & 0.3 & 0.74 & 0.74 & 0.74 & 0.78 & $0.39 *$ & $0.91 *$ \\
\hline 5.0 & 0.5 & 0.67 & 0.70 & 0.63 & 0.67 & 0.43 & 0.77 \\
\hline 5.0 & 1.0 & 0.68 & 0.79 & 0.51 & 0.65 & 0.46 & 0.73 \\
\hline 5.0 & 2.0 & 0.80 & 0.98 & 0.36 & 0.66 & 0.47 & 0.76 \\
\hline 5.0 & 5.0 & 1.00 & 1.00 & 0.28 & 0.78 & 0.40 & 1.00 \\
\hline 5.0 & 10.0 & 1.00 & 1.00 & 0.36 & $0.98 *$ & 0.41 & - \\
\hline 1.0 & 0.1 & 0.73 & 0.76 & 0.68 & $0.91 *$ & $0.42 *$ & $1.00 *$ \\
\hline 1.0 & 0.3 & 0.82 & 0.97 & 0.43 & 0.90 & 0.43 & 1.00 \\
\hline 1.0 & 0.5 & 0.94 & 1.00 & 0.40 & 0.91 & $0.42 *$ & 1.00 \\
\hline 1.0 & 1.0 & 1.00 & 1.00 & 0.39 & 1.12 & $0.33^{*}$ & - \\
\hline 1.0 & 2.0 & 1.00 & 1.00 & 0.37 & 0.96 & $0.36^{*}$ & - \\
\hline 0.5 & 0.1 & 0.74 & 0.84 & 0.54 & $1.00 *$ & $0.28 *$ & - \\
\hline 0.5 & 0.3 & 0.97 & 1.00 & 0.41 & $1.00 *$ & 0.23 & - \\
\hline 0.5 & 0.5 & 1.00 & 1.00 & 0.40 & - & - & - \\
\hline 0.5 & 1.0 & 1.00 & 1.00 & 0.33 & - & $0.11 *$ & - \\
\hline 0.5 & 2.0 & 1.00 & 1.00 & 0.46 & $1.00 *$ & $0.15^{*}$ & - \\
\hline 0.1 & 0.1 & 1.00 & 1.00 & 0.46 & - & - & - \\
\hline 0.1 & 0.3 & 1.00 & 1.00 & 0.37 & - & $0.05^{*}$ & - \\
\hline 0.1 & 0.5 & 1.00 & 1.00 & 0.45 & $1.00 *$ & 0.16 & - \\
\hline 0.1 & 1.0 & 1.00 & 1.00 & 0.41 & $1.00 *$ & 0.22 & - \\
\hline
\end{tabular}


at the wall is different from that in the bulk but with a reverse trend in contrast to its longitudinal component. For example, at the temperature $T=10.0$, the magnitude of the exponent at the wall $\nu_{y}$ varies substantially with the field $\left(\nu_{y} \simeq 0.7-1.0\right)$; it shows a nonmonotonic dependence with a minimum, unlike corresponding longitudinal value $\left(\nu_{x}\right)$ at the wall but somewhat similar to $\nu_{x}$ in the bulk [see Fig. 4]. An analogous trend is also seen for the transverse value of the exponent $\nu_{y}$ in the bulk. A detailed estimate of these exponents at various values of $B$ in a range of temperature $T=0.1-100.0$ is presented in Table I. We see that these exponents also depend on the temperature. For example, at $B=1.0, \nu_{x}$ in bulk ( $\nu_{y}$ at the wall) increases from about $0.7(0.8)$ on reducing the temperature from $T=100.0$ to 1.0; i.e., the field becomes more effective at low temperatures. The magnitude of the exponent $\nu$ for the radius of gyration $\left(R_{g}\right)$ has the same order of value both at the wall and in the bulk (2/3 at high $T$ to 1 at low $T$ ). The role of the $x$ and $y$ components in the bulk are reversed from that at the adsorbing wall which is consistent with previous simulations in different systems $[15,25]$. Thus, we find that for our model (1) the power-law scaling exponent for both the longitudinal and the transverse spread of the radius of gyration is nonuniversal as it depends on the field strength and temperature. (2) The crossover in scaling of the longitudinal component of the radius of gyration from bulk to wall is opposite to that for the transverse spread. The details including the effects of the wall interaction on profile and scaling will be published elsewhere.

This work is supported by a NSF-EPSCoR grant. G.M.F. acknowledges support of computer time at the CADCAM and Computer Centre of the National University of Singapore.

[1] M. Kardar, G. Parisi, and Y.C. Zhang, Phys. Rev. Lett. 56, 889 (1986).

[2] M. Kardar and Y.C. Zhang, Phys. Rev. Lett. 58, 2087 (1987).
[3] Dynamics of Fractal Surfaces, edited by F. Family and T. Vicsek (World Scientific, Singapore, 1991).

[4] A.L. Barabasi and H.E. Stanley, Fractal Concepts in Surface Growth (Cambridge University Press, Cambridge, 1995).

[5] R.P. Wool, Polymer Surfaces: Structure and Strength (Hanser Press, New York, 1995).

[6] D. A. Huse, C. L. Henley, and D. S. Fisher, Phys. Rev. Lett. 55, 2924 (1985).

[7] D. Forster, D. R. Nelson, and M. J. Stephen, Phys. Rev. A 16, 732 (1977).

[8] G. M. Foo and R. B. Pandey, J. Chem. Phys. (to be published).

[9] G. J. Fleer et al., Polymers at Interfaces (Chapman and Hall, London, 1993).

[10] E. Eisenriegler, Polymers near Surfaces (World Scientific, Singapore, 1993).

[11] S. A. Safran, Statistical Thermodynamics of Surfaces, Interfaces, and Membranes (Addison-Wesley, Reading, MA, 1994).

[12] G. S. Grest, Phys. Rev. Lett. 76, 4979 (1996).

[13] U. D’Ortona, J. D. Coninck, J. Koplik, and J. R. Banavar, Phys. Rev. Lett. 74, 928 (1995).

[14] R. Zajac and A. Chakrabarti, J. Chem. Phys. 104, 2418 (1996).

[15] P. Y. Lai, J. Chem. Phys. 103, 5742 (1995).

[16] S. K. Kumar, M. Vacatello, and D. Y. Yoon, J. Chem. Phys. 89, 5206 (1988).

[17] C. Wu et al., Electrophoresis 17, 1103 (1996).

[18] D. A. Hoagland, D. L. Smisek, and D. Y. Chen, Electrophoresis 17, 1151 (1996).

[19] G. W. Slater and J. Noolandi, Biopolymers 28, 1781 (1989).

[20] J. M. Deutsch, Science 240, 922 (1988).

[21] E. O. Shaffer and M. O. de la Cruz, Macromolecules 22, 1351 (1989).

[22] L.S. Lerman and H.L. Frisch, Biopolymers 21, 995 (1982).

[23] O. J. Lumpkin, P. Djardin, and B. H. Zimm, Biopolymers 24, 1573 (1985).

[24] J. Noolandi et al., Phys. Rev. Lett. 58, 2428 (1987).

[25] J. Baschnagel and K. Binder, Macromolecules 28, 6808 (1995). 